American Journal of Applied Sciences 5 (6): 665-671, 2008

ISSN 1546-9239

(C) 2008 Science Publications

\title{
A Novel Teletaction Control System for Detection of Human Pulse Wave with Applications in Teleoperations
}

\author{
${ }^{1}$ Javad Dargahi, ${ }^{1}$ Saeed Sokhanvar, ${ }^{2}$ Siamak Najarian, and ${ }^{3}$ Farhad Tabatabai Ghomshe \\ ${ }^{1}$ Department of Mechanical and Industrial Eng., Concordia University, \\ 1455 de Maisonneuve Blvd. West, Montreal, Quebec, Canada \\ ${ }^{2}$ Artificial Tactile Sensing and Robotic Surgery Lab, Department of Biomechanics, \\ Faculty of Biomedical Engineering, Amirkabir University of Technology, Tehran, Iran \\ ${ }^{3}$ University of Social Welfare and Rehabilitation Sciences, Tehran, Iran
}

\begin{abstract}
The design of a smart medical tool that can detect the human pulse and transfer its data to a remote location is presented. The resulting control system can be used to detect the pulse wave of human obtained from the wrist area. Fabrication of the tactile sensor and its calibration procedure are discussed as well. Additionally, the design of the pulse display system and that of its control system along with the position feedback control are accomplished. The experimental results of the position feedback control system are compared with the modeling and good agreement is obtained.
\end{abstract}

Key words: Tactile sensing, teletaction, pulse detection, feedback control

\section{INTRODUCTION}

Despite a lot of research activities, robot-assisted surgery is still in its infancy ${ }^{[1-7]}$. Developing teletaction, i.e., long-distance feel, has proven to be a complex process. As a result of this, the current prototypes are somewhat away from functioning at a useful level that can be comfortably used by the surgeons located far away from the operating site ${ }^{[8-12]}$. In telerobotic surgery, an expert surgeon could be sitting in his/her office, while operating on a patient far away from his/her location.

Using teletaction, information about texture, local compliance, and local shape, which are important in applications such as telesurgery or handling of fragile objects in telerobotics, can be obtained ${ }^{[13-15]}$. A teletaction system is in effect, a system that senses tactile information from the environment and displays that tactile information to a remote operator. One possible application is on a robotic laparoscopic telesurgery system in which the tactile sensor is mounted on the laparoscopic instrument and the tactile display is mounted on the user interface ${ }^{[16-18]}$. The ideal system would be the one by using which the sensations felt by the operator would be indistinguishable from direct contact with the sensed object or tissue. Teletaction systems normally consist of a tactile sensor, a tactile data processor with feedback control capabilities, and a tactile display system $^{[19-21]}$.

From a biomedical engineering point of view, teletaction is the sensing of a remote biological tissue to transmit cutaneous information (tactile sense) from a remote tactile sensor to an operator's skin (typically the fingertips ${ }^{[22,23]}$. In the medical areas, the techniques have been used in computer-assisted surgery and virtual environments for training. This sense of touch is important in applications such as surgery, where the feel of the environment provides knowledge that cannot be obtained by purely visual means ${ }^{[24-26]}$. The tactile interface reproduces, as accurately as possible, the parameters such as force (static and dynamic), texture, roughness, temperature and shape ${ }^{[27]}$.

In a typical teletaction system, the sensed information is transformed to controlling data by the processing unit ${ }^{[28]}$. It controls the tactile display devices which are used to provide subjects with the sensation of touching objects directly with the skin.

By reviewing the literature of the techniques of teletaction system conducted over the past decade, it is concluded that there have been a growing number of applications in teletaction systems ${ }^{[29-31]}$. This includes text and graphics, medical applications, entertainment and educational applications, military applications, tactile displays embedded in consumer electronics and wearable devices and telerobotic systems.

Corresponding Author: Javad Dargahi, Department of Mechanical and Industrial Eng., Concordia University, 1455 de Maisonneuve Blvd. West, Montreal, Quebec, Canada 
Following the above-mentioned progress and our previous studies, the need for the design of a smart medical tool that can detect the human pulse and transfer its data to a remote location is of great importance. This research work focuses on the construction of a novel control system, which can be used to detect the pulse wave of human obtained from the wrist area.

\section{MATERIALS AND METHODS}

Fabrication of the sensor: All the parameters and specifications of the PVDF film (Good Fellow Company, USA) used in the experiment are listed in Table 1.

The first step is the essential work which is to join the PVDF sensing film to the connecting wire. The procedure is as follows:

1. Cutting the PVDF film in right shape and size which can be wrapped up on the semi-sphere tip of the Plexiglas probe and cleaning up the PVDF film by using industrial alcohol.

2. Using tape to affix the film on the working bench.

3. Cutting the copper wire to the required length.

4. Cleaning the copper wire and using tape to fix it on the bench with one end securely contacting the top surface of the film.

5. Using the conductive glue, and then affixing the positive electrode at the spot where the end of the copper wire contacts with the film.

6. Allowing it to dry for at least ten hours and, following steps 2-6, repeating the procedure for the negative electrode.

The next step was to cut a circular shape rubber slightly larger than the PVDF film and glue the rubber at the probe tip. Finally, we glued the wired PVDF film on the rubber surface with the positive and negative electrode connecting wire slightly hung over covered area.

Calibration of the sensor: In order to calibrate the sensor, an experimental set-up was built. This is shown in Fig. 1. In order to calibrate the sensor for display side, the sensor was placed on a micropositioner. A probe of $16 \mathrm{~mm}$ diameter was used to apply a sinusoidal load of $20 \mathrm{~Hz}$ via a vibrator. The micropositioner was used in order to ensure that the probe was positioned on the center of the sensor in a precise manner. The vibrator was activated by a power amplifier and a signal generator. The magnitude of the
Table 1: Parameters of PVDF used in modeling

\begin{tabular}{|c|c|c|c|}
\hline Name & Description & Value & Unit \\
\hline $\mathrm{g}_{31}$ & $\begin{array}{l}\text { Piezo coefficient in drawn } \\
\text { direction (Voltage Mode) }\end{array}$ & 0.15 & $\mathrm{Vm} \cdot \mathrm{N}^{-1}$ \\
\hline $\mathrm{g}_{32}$ & $\begin{array}{l}\text { Piezo coefficient in transverse } \\
\text { direction (Voltage Mode) }\end{array}$ & 0.15 & $\mathrm{Vm} \cdot \mathrm{N}^{-1}$ \\
\hline$d_{31}$ & $\begin{array}{l}\text { Piezo coefficient in drawn } \\
\text { direction (Charge Mode) }\end{array}$ & 18.00 & $\mathrm{pC} . \mathrm{N}^{-1}$ \\
\hline$d_{32}$ & $\begin{array}{l}\text { Piezo coefficient in transverse } \\
\text { direction (Charge Mode) }\end{array}$ & 2.00 & $\mathrm{pC} . \mathrm{N}^{-1}$ \\
\hline$E_{p v}$ & Tensile and transverse modulus & 2.20 & GPa \\
\hline$r_{\mathrm{pv} 1}$ & $\begin{array}{l}\text { Contacting radius of PVDF film } \\
\text { at pulse sensing side }\end{array}$ & 8.00 & $\mathrm{~mm}$ \\
\hline$r_{p v 2}$ & $\begin{array}{l}\text { Contacting radius of PVDF film } \\
\text { at pulse display side }\end{array}$ & 9.00 & $\mathrm{~mm}$ \\
\hline$d_{\mathrm{pv} 1}$ & $\begin{array}{l}\text { Thickness of PVDF film at pulse } \\
\text { sensing side }\end{array}$ & 0.025 & $\mathrm{~mm}$ \\
\hline$d_{\text {pve } 2}$ & $\begin{array}{l}\text { Thickness of PVDF film at pulse } \\
\text { display side }\end{array}$ & 0.025 & $\mathrm{~mm}$ \\
\hline
\end{tabular}

(a)
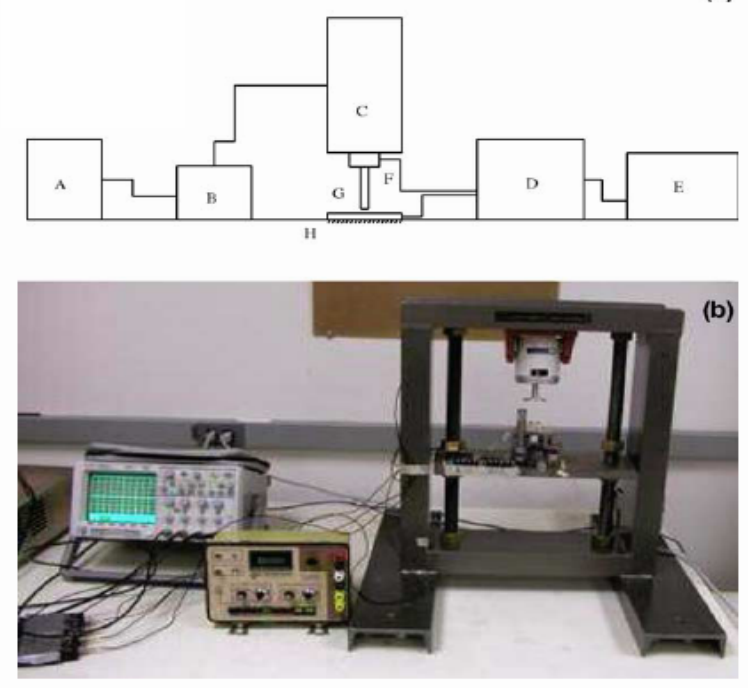

Fig. 1: Schematic (a) and experimental (b) set-up for the calibration of the sensors. In (a), we have: A-Power Amplifier, B-Signal Generator, CVibrator, D-Oscilloscope, E-Charge Amplifier, F-Force Transducer, G-Probe, and H-Tactile Sensor

applied load was measured by a force transducer which was placed between the probe and the vibrator. The output from both PVDF sensor and the force transducer was fed though a charge amplifier and the result was recorded on an oscilloscope.

Various magnitudes of loads were applied and the output from the PVDF sensor was recorded. The results show that the output voltages vary linearly with the applied loads. 
Design of pulse display system: In human tactile sensing system, SA II and FA II have larger receptive fields and only a single unit will respond to vibrations applied at any point on a finger. This suggests SA II and FA II unit responses do not localize vibratory stimulus on the skin surface. Therefore, a single vibration display for the fingertip to provide the pulse information has been provided for the pulse display system.

A linear actuator is used as driving unit which generates pulse load on a pulse display block. The pulse display block consists of a PVDF pulse display sensor film which conveys the force feedback to the control system and another rubber layer which is adopted as a simulation of human skin. The pulse vibration applies load on the rubber layer and develops stress and strain. Therefore, at the pulse display side, when the finger touches the top of the rubber surface, the same scenario is created at both pulse sensing and display sides as shown in Fig. 2.

\section{Design of control system for pulse display system:}

The step motor is an electromagnetic device that converts digital pulses into mechanical shaft rotation or linear movement. The advantages of step motors are the low cost, high reliability, high torque at low speeds and a simple, rugged construction that operates in almost any environment. The main disadvantage of a step motor is the resonance effect which often exhibits at low speeds and decreasing torque with increasing speed. A stepping motor system consists of three basic elements, indexer, driver and step motor. The indexer is a microprocessor (or controller) capable of

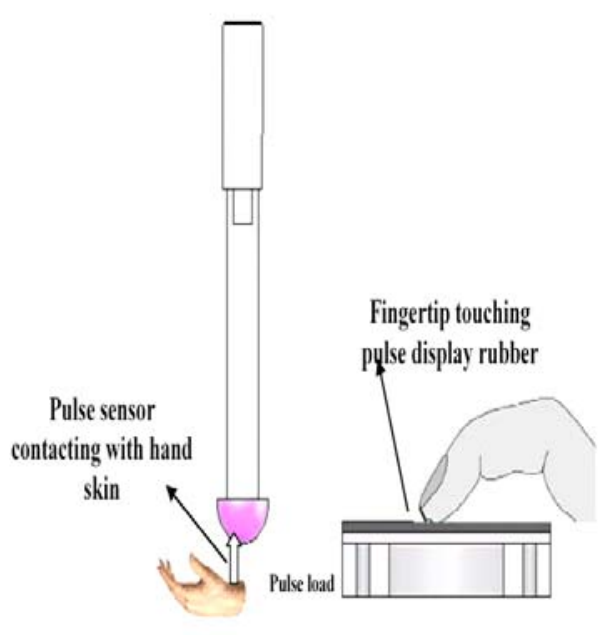

Fig. 2: Identical condition in pulse sensing and display generating step pulses and direction signals for the driver. In addition, the indexer is typically required to perform many other sophisticated command functions such as managing other control functions, including acceleration, deceleration, steps per second and distance. The indexer can also be interfaced and control many other external signals.

The driver (or amplifier) converts the indexer command signals into the power necessary to energize the motor windings. There are numerous types of drivers, with different current/amperage ratings and construction technology. Different motor needs specific drivers to operate, thus, the driver selection process is critical in the motion control system.

In the pulse display system, we designed a hybrid bi-polar driving stepping motor (KH42KM2-951) which was embedded in the linear actuator system. The number of phases is 2 and the step angle is $1.8^{\circ}$. In order to control the motion of the step motor to reproduce human pulse signal, the accurate micro step driver is necessary. In the experiment, a CENTENT micro step driver is applied for the step motor control. In this pulse display control system, a VCO (Voltage-Controlled-Oscillator) controller and a PID (Proportional-Integral-Derivative) controller are both used in the position feedback control system. These will be discussed in the next section.

PID and VCO controllers: The PID controller has the transfer function form as below:

$$
\frac{\mathrm{u}(\mathrm{s})}{\mathrm{E}(\mathrm{s})}=\mathrm{K}_{\mathrm{P}}+\frac{\mathrm{K}_{\mathrm{I}}}{\mathrm{s}}+\mathrm{K}_{\mathrm{D}} \mathrm{s}=\frac{\mathrm{K}_{\mathrm{D}} \mathrm{s}^{2}+\mathrm{K}_{\mathrm{P}} \mathrm{s}+\mathrm{K}_{\mathrm{I}}}{\mathrm{s}}
$$

where $\mathrm{u}(\mathrm{s})$ is the control signal, $\mathrm{E}(\mathrm{s})$ is the error between reference and controlled variable, $K_{p}$ is the proportional gain, $\mathrm{K}_{\mathrm{I}}$ is the integral gain and $\mathrm{K}_{\mathrm{D}}$ is the derivative gain.

Since the human pulse signal is a frequency altering signal, the step motor output frequency should be controlled to follow this change. Therefore, the VCO controller has been introduced to the stepping motor control system. The VCO block from MATLAB/SIMULINK is used for the step motor control.

The VCO block generates a signal $y(t)$ whose frequency shift from the oscillation frequency parameter is proportional to the input signal. The input signal is interpreted as a voltage. If the input signal is denoted as $\mathrm{u}(\mathrm{t})$, then the output signal is:

$$
y(t)=A_{c} \cos \left(2 \pi f_{c} t+2 \pi k_{c} \int_{0}^{t} u(\tau) d \tau+\varphi\right)
$$


where $A_{c}$ is the output amplitude parameter, $F_{c}$ is the oscillation frequency parameter, $\mathrm{K}_{\mathrm{c}}$ is the input sensitivity parameter and $\varphi$ is the initial phase parameter.

Position feedback control: In the close loop position feedback control system (shown in Fig. 3 and 4), the magnitude of human pulse signal sampled at the tactile sensor side is set as the desired (reference input) position signal. An optical incremental encoder inside the stepping motor counts the number of the step motor pulses which represent the displacement (position) of the actuator. The feedback displacement is sent back to the comparator. The error signal $e$ from the comparator is divided into two flows. One flow is sent to the direction control to decide the step motor direction. For example, if the error is greater than zero $(e>0)$, the direction is set to 1 ; if the error is less than zero $(e<0)$, the direction is set to 0 . This logic signal is sent to the direction control port in the step motor drive and to decide the linear actuator motion direction. Another flow of $e$ is sent to the PID controller, in which the control signal $u$ is calculated based on the PID parameters. The VCO controller calculates the output frequency of the step pulse based on the input frequency of $u$ and passes the pulses into the pulse port in the step motor drive. Therefore, based on the direction control signal and frequency-altering step pulses signal, the output position of linear actuator is controlled to track the position of the input signal.

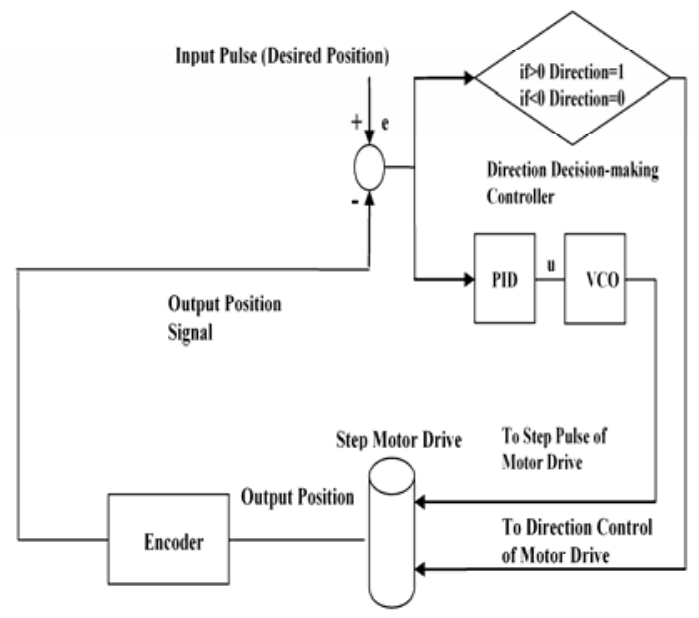

Fig. 3: Flow chart diagram of position feedback control system

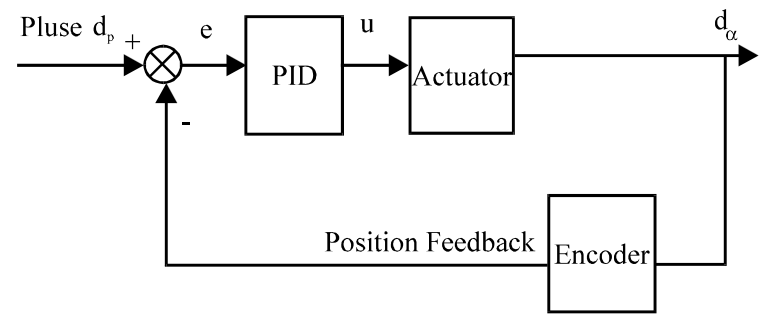

Fig. 4: Block diagram of position feedback control system

The position control has the advantage of easy implementation due to the built-in encoder. However, it is hard to obtain reproduced feeling. Hence, the purpose of the position feedback system in this research is to verify the experiment setup and identify the parameters of the actuator system.

\section{RESULTS AND DISCUSSION}

Modeling of the actuator system: In this pulse display system, the linear actuator system consists of step motor, encoder, drive and indexer (VCO). In order to find the entire actuator system model, the real time open loop control experiment method is used to identify the parameters and define the model as second order transfer function. In the real time experiment, a sinusoidal signal was used as the reference position input to activate the actuator plant and the position of the linear probe movement is recorded as the output of the actuator plant. Fig. 5 presents the experimental results including the actuator input and output data.

The solid curve is the input signal $\mathrm{x}_{0}(\mathrm{t})$ and the dashed curve is the output $x(t)$. The $X$-axis represents the sampling time. When the input signal is a sinusoid signal, it is noticed that the output signal is also a sinusoidal curve with a phase difference $\phi$. The actuator plant is assumed to be a second order system.

Based on the theory of vibration, the differential equation of the actuator system in free body equation of motion is expressed as:

$$
\mathrm{m} \ddot{\mathrm{x}}+\mathrm{c} \dot{\mathrm{x}}+\mathrm{kx}=\mathrm{X}_{0} \sin \omega \mathrm{t}
$$

where $\mathrm{m}$ is the mass of actuator, c is damping coefficient and $\mathrm{k}$ is stiffness coefficient. $\mathrm{X}_{0}$ is the amplitude of the input signal. When the input is a harmonic signal, $X_{0} \sin \omega t$, the solution to the equation consists of two parts, the complementary function, which is the solution of the homogeneous equation, and the particular integral. The particular solution is a 




Fig. 5: Experiment results of open-loop position control (actuator identification)

steady-state output oscillation of the same frequency $\varpi$ as that of the excitation. The particular solution can be written as:

$$
\mathrm{x}=\mathrm{X} \sin (\varpi \mathrm{t}-\phi)
$$

where $\mathrm{X}$ is the amplitude of the output oscillation and $\phi$ is the phase of the output with respect to the input $\mathrm{X}_{0}$. Hence, the following equations are obtained:

$$
\begin{gathered}
\ddot{x}=-X \varpi^{2} \sin (\varpi t-\phi) \\
\dot{x}=X \varpi \cos (\varpi t-\phi)
\end{gathered}
$$

The amplitude and phase are found as below:

$$
\frac{X}{X_{0}}=\frac{1}{\sqrt{\left(k-m \varpi^{2}\right)^{2}+\left(c \varpi^{2}\right)^{2}}}
$$

The phase difference is obtained as:

$$
\phi=\tan ^{-1} \frac{\mathrm{c} \varpi}{\mathrm{k}-\mathrm{m} \varpi^{2}}
$$

From Figure 5, the phase $\phi$ can be identified as:

$$
\sin \phi=18 / 30=3 / 5 \Rightarrow \tan \phi=3 / 4
$$

and

$$
\varpi=2 \pi \mathrm{f}=2 \pi / \mathrm{T}=\frac{2 \pi}{\mathrm{t}_{\mathrm{s}} \times \mathrm{N}}
$$

where $t_{s}$ is the sampling period and $\mathrm{N}$ is the number of samples in one period of the input signal. $f$ and $T$ are the oscillation frequency and period, respectively. According to the sampling theorem, for a limited bandwidth (band-limited) signal with maximum frequency $f_{\max }$, the equally-spaced sampling frequency $f_{s}$ must be greater than twice of the maximum frequency $\mathrm{f}_{\text {max }}$. In this system, the maximum frequency of the pulse is $50 \mathrm{~Hz}$. Therefore, the sampling frequency in this system must be larger than $100 \mathrm{~Hz}$. In the experiment, the sampling interval is set as $\mathrm{t}_{\mathrm{S}}=0.0005$ second. The number of sampling in one period is $\mathrm{N}=0.4 \times 10^{4}$. Thus, one obtains $=3.14 \mathrm{rad} / \mathrm{s}$. The parameters of step motor and the linear actuator are listed in Tables 2 and 3.

By re-examining Fig. 5, we have the output amplitude $X=25$ and input force $X_{0}=150$ and from Table 2, the mass of the actuator system can be obtained as $\mathrm{m}=0.5 \mathrm{~kg}$. Hence, solving Eq. 7 and 8 , one can obtain the stiffness coefficient $\mathrm{k}$ and damping coefficient $\mathrm{c}$ as $\mathrm{k}=7.27$ and $\mathrm{c}=0.56$. The actuator is re-written in transfer function form as:

$$
\mathrm{G}(\mathrm{s})=\frac{\mathrm{F}_{\mathrm{a}}}{\mathrm{u}}=\frac{1}{\mathrm{~ms}^{2}+\mathrm{cs}+\mathrm{k}}
$$

From the above formulation, the transfer function of the actuator system is obtained as:

$$
G(s)=\frac{1}{0.5 s^{2}+0.56 s+7.27}
$$

Table 2: Linear actuator (Dyadic SCN-010-AS) parameters

\begin{tabular}{lcr}
\hline Name & Value & Unit \\
\hline Stroke & 0.100 & $\mathrm{~m}$ \\
Max. Thrust & $100 / 10.2$ & $(\mathrm{~N}) /(\mathrm{kgf})$ \\
Load Capacity & $5 \sim 15$ & $\mathrm{~N}$ \\
Max. Speed & 0.400 & $\mathrm{~m} / \mathrm{s}$ \\
Rod Diameter & 0.016 & $\mathrm{~m}$ \\
Weights & 1.000 & $\mathrm{~kg}$ \\
\hline
\end{tabular}

Table 3: Stepping motor (JAPAN SERVO KH42KM2) parameters

\begin{tabular}{lll}
\hline Name & Value & Unit \\
\hline Drive Method & Bi-Polar & - \\
Number of Phases & 2 & $\overline{\text { deg./step }}$ \\
Step Angle & 1.8 & $\mathrm{~kg}$ \\
Weights & 0.35 & \\
\hline
\end{tabular}


Table 4: PID parameters in position feedback control

\begin{tabular}{lrr}
\hline Name & Description & Value \\
\hline $\mathrm{K}_{\mathrm{P}}$ & Proportional & 13.00 \\
$\mathrm{~K}_{\mathrm{I}}$ & Integral & 0.20 \\
$\mathrm{~K}_{\mathrm{D}}$ & Derivative & 0.08 \\
\hline
\end{tabular}

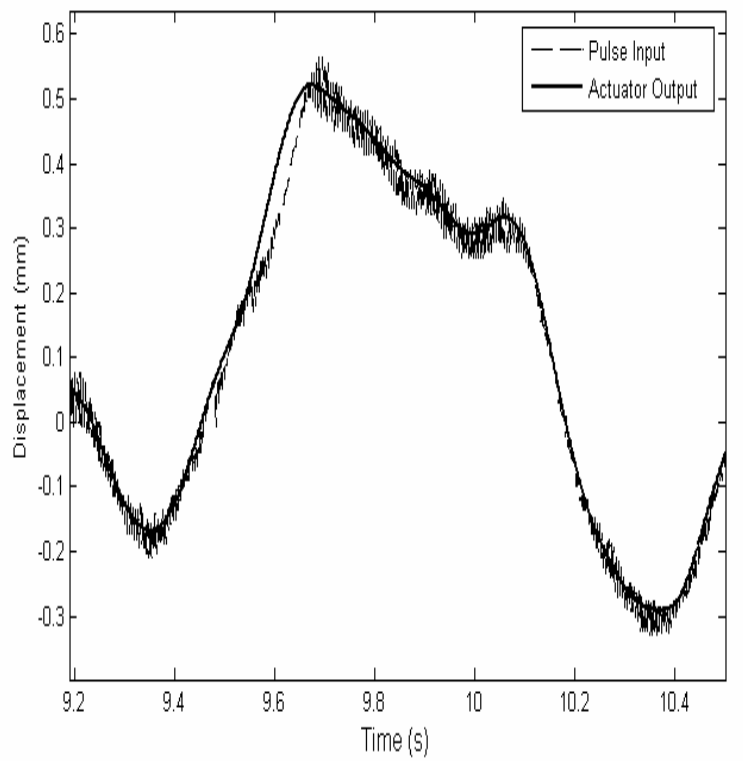

Fig. 6: 9.2 10.5 second sampled position control results

Experimental results of position feedback control The results of position control (with the measured reference human pulse) are shown in Fig. 6.

The parameters of PID controller are tuned by trialand-error and listed in Table 4.

Based on the position control results, the actuator output replicated the input pulse position to a great extent. The RMSE for the position feedback control experiment (during 10 second sample) is:

$$
\operatorname{RMSE}_{\mathrm{P}}=\sqrt{\frac{1}{\mathrm{~N}} \sum_{\mathrm{i}=1}^{\mathrm{N}} \mathrm{e}_{\mathrm{P}}^{2}}=0.0304
$$

where $e_{\mathrm{P}}$ is the position error between the output and input.

\section{ACKNOWLEDGEMENTS}

The authors thank the Institute for Robotics and Intelligent Systems (IRIS), and the Natural Sciences and Engineering Research Council (NSERC) of Canada for providing partial financial support. We also would like to express our gratitude to the Center of Excellence of Biomedical Engineering of Iran based in Amirkabir University of technology, Faculty of Biomedical Engineering for its contribution.

\section{REFERENCES}

1. Dario, P., 1991. Tactile Sensing-Technology and Applications. Sensors and Actuators A-Physical, 26: 251-261.

2. Dargahi, J. and S. Najarian, 2004. Analysis of a Membrane Type Polymeric-Based Tactile Sensor for Biomedical and Medical Robotic Applications. Sensors and Materials, 16: 25-41.

3. Dargahi, J. and S. Najarian, 2004. An Integrated Force-Position Tactile Sensor for Improving Diagnostic and Therapeutic Endoscopic Surgery. Bio-Medical Materials and Engineering, 14: 151-166.

4. Dargahi, J., 1998. Piezoelectric and Pyroelectric Transient Signal Analysis for Detecting the Temperature of an Object for Robotic Tactile Sensing. Sensor and Actuators A-Physical, 71: 89-97.

5. Dargahi, J. and S. Najarian, 2004. Theoretical and Experimental Analysis of a Piezoelectric Tactile Sensor for Use in Endoscopic Surgery. Sensor Review, 24: 74-83.

6. Fischer, H., B. Neisius and R. Trapp, 1995. Interactive Technology and a New Paradigm for Health Care. IOS Press, The Netherlands.

7. Howe, R.D., W.J. Peine, D.A. Kontarinis and J.S. Son, 1994. Remote Palpation Technology. Proceedings of IEEE Engineering in Medicine and Biology Magazine, 14: 318-323.

8. Bicchi, A., G. Canepa, D.De. Rossi, P. Iacconi and E.P. Scilingo, 1996. A Sensorized Minimally Invasive Surgery Tool for Detecting Tissue Elastic Properties. Proceedings of IEEE International Conference Robotics and Automation, Minneapolis, USA, 884-888.

9. Brouwer, I., J. Ustin, L. Bentley, A. Sherman, N. Dhruv and F. Tendick, 2001. Measuring in vivo Animal Soft Tissue Properties for Haptic Modeling in Surgical Simulation. In Studies in Health Technology Informatics-Medicine Meets Virtual Reality, Amsterdam: ISO Press, 69-74.

10. Dargahi, J. and S. Najarian, 2004. A Supported Membrane Type Sensor for Medical Tactile Mapping. Sensor Review, 24: 284-297. 
11. Hannaford, B., J. Trujillo, M. Sinanan, M. Moreyra, J. Rosen, J. Brown, R. Leuschke and M. MacFarlane, 1998. Computerized Endoscopic Surgical Grasper. In Studies in Health Technology Informatics-Medicine Meets Virtual Reality, Amsterdam, ISO Press, 265-271.

12. Dargahi, J. and S. Najarian, 2004. Human Tactile Perception as a Standard for Artificial Tactile Sensing-a review. International Journal of Medical Robotics and Computer Assisted Surgery, 1: 23-35.

13. Dargahi, J. and S. Najarian, 2005. Advances in Tactile Sensors Design/Manufacturing and Its Impact on Robotics Applications- a review. Industrial Robot, 32: 268-281.

14. Dargahi, J. and S. Najarian, 2003. An Endoscopic Force Position Grasper with Minimum Sensors. Canadian J. Elect. and Comp. Eng., 28: 155-161.

15. Dargahi, J., S. Najarian and X.Z. Zheng, 2005. Measurements and Modeling of Compliance Using a Novel Multi-Sensor Endoscopic Grasper Device. Sensors and Materials, 17: 7-20.

16. Dario, P., M.C. Carrozza, L. Lencioni, B. Magnani and S. D Attanasio, 1997. A Micro Robotic System for Colonoscopy. Proc. IEEE Int. Conf. on Robotics and Automation, IEEE Robotics and Automation Society, 1567-1572.

17. Lee, M.H. and H.R. Nicholls, 1999. Tactile Sensing for Mechatronics-A State-of-the-Art Surgery. Mechatronics, 9: 1-31.

18. Bar-Cohen, Y., C. Mavroidis, M. Bouzit, B. Dolgin, D. Harm, G. Kopchok and R. White, 2000. Virtual Reality Robotic Operation Simulations Using MEMICA Haptic System. Proc. Int. Conf. for Smart Systems and Robotics for Medicine and Space Applications, Houston, USA.

19. Fisch, A., C. Mavroidis, J. Melli-Huber and Y. Bar-Cohen, 2003. Haptic Devices for Virtual Reality, Telepresence, and Human-Assistive Robotics. chap. 4 in Biologically-Inspired Intelligent Robots, Bellingham, Wash.

20. Dargahi, J., M. Parameswaran and S. Payandeh, 2000. A Micromachined Piezoelectric Tactile Sensor for Endoscopic Grasper-Theory, Fabrication, and Experiments. J. Microelect. Sys., 9: 329-335.

21. Dargahi, J., 1993. The Application of Polyvinylidene Fluoride as a Robotic Tactile Sensor. Ph.D. dissertation, Glasgow Caledonian University, Glasgow, Scotland.
22. Fearing, R.S., G. Moy and E. Tan, 1997. Some Basic Issues in Teletaction. Proceedings of IEEE International Conference on Robotics and Automation, Albuquerque, USA, 3093-3099.

23. Dargahi, J., 2000. A Three Sensing Element Piezoelectric Tactile Sensor for Robotic and Prosthetic Applications. Sensors and Actuators A-Physical, 80: 23-30.

24. Dargahi, J., 2002. An Endoscopic and Robotic Tooth-Like Compliance and Roughness Tactile Sensor. J. Mech. Des., 124: 576-582.

25. Geisthoff, W., S.H. Tretbar, P.A. Federspil and P.K. Plinkert, 2004. Improved Ultrasound-Based Navigation for Robotic Drilling at the Lateral Skull Base. Int. Cong. Ser., 1268: 662-666.

26. Mirbagheri, A., J. Dargahi, S. Najarian and F. Tabatabai Ghomshe, 2007. Design, Fabrication and Testing of a Membrane Piezoelectric Tactile Sensor with Four Sensing Elements. Am. J. Applied Sci., 4: 645-652.

27. Mirbagheri, A., J. Dargahi, S. Najarian and F. Tabatabai Ghomshe, 2007. Orientation Detection of Sensed Objects using a Four Sensor Tactile System with Applications in Bio-robotics. Am. J. Applied Sci., 4: 925-931.

28. Darb Emamieh, G., A. Ameri, S. Najarian and A. Tavakoli Golpaygani, 2008. Experimental and Theoretical Analysis of a Novel Flexible Membrane Tactile Sensor. Am. J. Applied Sci., 5: 122-128.

29. Tavakoli Golpaygani, A., S. Najarian, M.M. Movahedi and G. Darb Emamieh, 2008. Fabrication of a Capacitance-based Tactile Sensor with Biomedical Applications. Am. J. Applied Sci, 5: 129-135.

30. Abouei Mehrizi, A., S. Najarian, M. Moini and F. Tabatabai Ghomshe, 2008. Tactile Distinction of an Artery and a Tumor in a Soft Tissue by Finite Element Method. Am. J. Applied Sci, 5: 83-88.

31. Dargahi, J., S. Najarian, R. Ramezanifard and F. Tabatabai Ghomshe, 2007. Fabrication and Testing of a Medical Surgical Instrument Capable of Detecting Simulated Embedded Lumps. Am. J. Applied Sci., 4: 957-964. 